\title{
Wolfgang Kühnel \\ Scheinbar konfliktfrei aneinander vorbei. \\ Eine Retrospektive auf die Generationsbeziehungen in den achtziger Jahren in der DDR
}

Zusammenfassung Die seit den siebziger Jahren immer offensichtlicher werdenden gesellschaftlichen Krisenprozesse in der DDR haben auch entscheidende Veränderungen in den Lebenszusammenhängen der Heranwachsenden und im Verhältnis zu vorangegangenen Generationen eingeleitet. Infolge der zunehmenden Durchstaatlichung ihrer Lebensverhältnisse wurde für die Jugendlichen immere mehr die Erfahrung sozialer Schließungen in ihren Bildungs- und Berufschancen, begleitet von einem Legitimationsverfall schulischer Lernangebote und politischer Partizipationsmöglichkeiten, bestimmend. Andererseits fand gegenläufig dazu ein Wandel in den soziokulturell und medial vermittelten Lernprozessen statt, der als »nischenhafte Modernisierung des Alltagskulturellen aufgefaßt werden kann. Dieser Prozeß hat ebenso eine gewisse Erosion in den traditionellen Autoritätsverhältnissen und Erfahrungsbezügen zur Erwachsenengeneration begünstigt.

Die Bedeutung des Generationsproblems werde immer dann ersichtlich, »sobald es sich um das genauere Verständnis der beschleunigten Umwälzungserscheinungen der umittelbaren Gegenwart handelt. (Mannheim 1970, S. 522) Von diesem Grundverständnis aus entwickelte Karl Mannheim in den bewegten zwanziger Jahren ein Generationskonzept, mit dem sich seither auch immer wieder aufs neue Generationen von Sozialwissenschaftlern beschäftigt haben. Und wieder leben wir in einer historischen Phase, wo die dramatischen Umbrüche in den osteuropäischen Gesellschaften, so auch in der DDR, nach Erklärungen suchen. Wer waren die Akteure des Wandels in der DDR und wie deuteten sich die Veränderungen womöglich im Wechsel generativer Lebens- und Erfahrungszusammenhänge an?

Die polnischen Soziologen scheinen es in diesem Punkt etwas einfacher zu haben als wir. So konnte Adamski (1982, S. 54) bereits Ende der siebziger Jahre nachweisen, daß die krisenhaften Transformationsprozesse in der polnischen Gesellschaft in sehr starkem Maße durch den Eintritt jüngerer Generationen in die Geschichte unterstützt wurden. Infolgedessen sei es zu einer sichtlichen Beschleunigung des Wandels in den kulturellen Beziehungen und im sozio-politischen System gekommen. Adamski geht sogar soweit, von einer $\gg$ Juventisation« der polnischen Gesellschaft (vgl. Adamski 1982) zu sprechen.

So einfach stellt sich das in der DDR allerdings nicht dar. Zwar gibt es bereits erste Anhaltspunkte dafür, daß ein Großteil derjenigen, die im Frühherbst des vergangenen 
Jahres in die Bundesrepublik ausgereist sind, junge Facharbeiter waren (vgl. Mitter/ Wolle 1990, S. 85). Für die daheim gebliebenen Protestierenden lassen sich solche eindeutigen Belege jedoch nicht finden. Beispielsweise konnten in den ersten Untersuchungen der Montagsdemonstrationen in Leipzig kaum nennenswerte Unterschiede der Demonstranten nach dem Alter nachgewiesen werden (vgl. Mühler/Wilsdorf 1990, S. 7). Dies besagt natürlich wenig, solange keine aussagefähigen Untersuchungen zur Hand sind. Aber immerhin spricht einiges dafür, daß sich im Zuge des Umbruchs in der DDR ein Wechsel der Akteure und Generationen vollzogen haben muß (vgl. Matthes/Müller-Hartmann 1990).

Die Plötzlichkeit und die ausgesprochene Dramatik des Wandels in der DDR läßt oftmals vergessen, daß die Spuren der Veränderungen, besonders in den Lebenszusammenhängen Heranwachsender, bis in die späten siebziger Jahre zurückverfolgt werden können.

Gegenïber vorangegangenen Generationen wurde für die Jugendlichen immer mehr die Erfahrung sozialer Schließungen in den Bildungs- und Berufschancen, bei einem gleichzeitigen Legitimationsverfall schulischer Lernangebote wie auch politischer Partizipationsmöglichkeiten im Rahmen der staatlichen Jugendorganisation bestimmend. Entkoppelt von den institutionalisierten, verstaatlichten Sozialisationsprozessen, ja man möchte sogar meinen, gegenläufig dazu fand andererseits in begrenztem Maße ein Wandel in den soziokulturellen und medial vermittelten Lernprozessen Jugendlicher statt, der in gewisser Weise als alltagskulturelle Modernisierung begriffen werden kann. Damit war gleichermaßen eine Erosion in den Autoritätsverhältnissen zu den Älteren und eine Entwertung der vor allem in der Schule offiziell vermittelten Wissens- und Traditionsbeständen verbunden. Die Modernisierung des Lebenszusammenhanges Jugendlicher ist zwar generell ein Zeichen für den Strukturumbruch von traditionellen Industriegesellschaften. Im Unterschied dazu verdankt sie ihre Existenz in der DDR der Tatsache, daß das vom staatlich-administrativen System ausgehende Bestreben, alle Lebensvollzüge dementsprechenden normativen Regelungen zu unterwerfen, immer mehr an Grenzen gestoßen ist.

Eine sich demgegenüber sehr viel rascher verändernde alltägliche Lebenswelt der Jugendlichen, hervorgerufen durch Internationalisierungstendenzen im Freizeit-, Medien- und Konsumbereich, wurde zusehends zu einer, immerhin offiziell tolerierten, aber alles in allem unberechenbaren »Restgröße «. Insofern mußten die alltagskulturellen Erfahrungszusammenhänge immer mehr mit den blockierten Entwicklungsmöglichkeiten in Beruf, Schule und Politik kollidieren.

\section{Der Wandel historisch-struktureller Rahmenbedingungen in der DDR-Gesellschaft als Voraussetzung für die Herausbildung unterschiedlicher generativer Lebenszusammenhänge}

Wohl besteht der kleinste gemeinsame Nenner all jener sozialwissenschaftlichen Analysen, in denen das Generationskonzept favorisiert wird, in der Annahme, damit 
gesellschaftliche Wandlungsprozesse erklären zu können. Doch wie das geschehen soll und welche Erklärungskraft man dem Generationsansatz überhaupt zubilligt, darüber gibt es recht unterschiedliche Auffassungen.

Mitunter gewinnt man den Eindruck, daß die bereits von Mannheim (1970, S. $509 \mathrm{ff}$. und S. 514 ff.) herauspräparierte Differenzierungslinie zwischen eineseits »quantifizierbaren « und andererseits »qualifizierbaren « Zugriffen auf das Generationsproblem in anderer Weise auch heute noch fortbesteht. So sehen vor allem die Vertreter der Sozialstruktur- und Mobilitätsforschung im Ansatz der Kohortenanalyse einen Beitrag zur Klärung des Verhältnisses zwischen gesellschaftlichem Wandel und Lebensverlaufswandel auf Seiten der Individuen (vgl. Mayer 1980, S. 5). In diesem Zusammenhang wird der Generationsbegriff entweder mit dem der Kohorte gleichgesetzt oder aber auf das Generationskonzept verzichtet, unter Hinweis darauf, daß die Annahme einer homogenen Erlebnis- und Erfahrungsgemeinschaft von Altersgruppen in keinem Fall zutreffen würde. Auf der anderen Seite stehen eher deskriptive Erklärungen, wo Generationstypologien auf der Grundlage der für bestimmte Altersgruppen prägenden zeitgeschichtlichen Erlebnisse und Strebungen entworfen werden. Wahrscheinlich ließe sich Schelskys (1957) »Skeptische Generation « in diese Tradition ebensogut einordnen wie Zinneckers und Fuchs' Generationsentwurf der »Kinder des Wachstums,..., als Jugend des Jugendprotests der 80er Jahre und der neuen sozialen Bewegungen « (Fuchs 1986, S. 137). Mag man noch so viele Einwände gegen das Mannheim'sche Generationskonzept vorbringen', so scheint jedenfalls bislang eine tragfähige Verbindung zwischen den beiden analytischen Strängen nicht in Sicht zu sein.

Nun hat man gut reden, wenn man aus der Perspektive des Standes sozialwissenschaftlicher Forschungen in der DDR den gesellschaftlichen Wandel nach dem darin enthaltenen Wandel von generativen Lebenszusammenhängen befragt. Außer einigen wenigen Untersuchungen zur sozialstrukturellen Entwicklung ${ }^{2}$ und Forschungen über den Einstellungswandel ${ }^{3}$ Jugendlicher gibt es bislang keine historisch-vergleichenden Analysen oder gar Replikationsstudien, woraus man Anhaltspunkte darüber gewinnen könnte, in welcher Weise die gesellschaftliche Entwicklung in der DDR von unterschiedlichen Generationen erfahren und bewältigt wurde. Selbst für den von mir vorgelegten Ansatz, Generationen als Organisationsformen der gesellschaftlichhistorischen Regelung von Zeitlichkeit sozialer Gruppen und Individuen zu fassen (vgl. Kühnel 1987), lassen sich nur sehr vage empirische Belege für den generativen Wandel in der DDR beibringen. Der einzig mögliche Zugang, der sich dazu anbietet, ist, die sozialstrukturelle Entwicklung zu unterschiedlichen Zeiträumen in ihrer Bedeutung für die Konstitution verschiedener generativer Handlungs- und Erfahrungsbedingungen zu rekonstruieren.

Stark vergröbert lassen sich drei Phasen unterscheiden, in deren Verlauf sich die Lebenszusammenhänge des Nachwuchses sozialer Gruppen auch sehr verschieden und ungleichzeitig verändert haben. Dabei gilt es zu berücksichtigen, daß der soziale Wandel in der DDR von Anfang an durch politische Eingriffe von Partei und Staat geprägt war. Das hat dazu beigetragen, die Entwicklung der Eigentums-, Bildungs- 
und Qualifikationsstrukturen sowie der Beschäftigungs- und Einkommensverhältnisse stets zentralistisch zu steuern.

Bis Anfang der sechziger Jahre war die Herausbildung der sozialen Grundstruktur im wesentlichen abgeschlossen. Die Aufbauphase in den vierziger und fünfziger Jahren eröffnete für die verschiedenen sozialen Gruppen einen Prozeß grundlegender Umgestaltung, der hauptsächlich zu einem Anwachsen der Beschäftigten in Arbeiterberufen und in der Ländwirtschaft führte. Dabei nahm die Anzahl der in der Großindustrie beschäftigten Arbeiter am schnellsten zu (vgl. Dittrich 1981, S. 256). Die hauptsächlichen Quellen des Zustroms waren Frauen aus der nichtarbeitenden Bevölkerung, Jugendliche und Arbeitslose. Die Aufnahme von Beschäftigungsverhältnissen in der staatlichen Industrie wurden gefördert durch Lohnanreize und verbesserte Arbeitsbedingungen. Die Aussicht, eine Veränderung der Lebenssituation durch Qualifikation zu erreichen, spielte nur für einen kleinen Teil der Jugendlichen dieser sozialen Gruppe eine Rolle. Die Landbevölkerung, bis Anfang der sechziger Jahre endgültig in landwirtschaftlichen Produktionsgenossenschaften organisiert, rekrutierte sich zum größten Teil aus ehemaligen Klein- und Mittelbauern, Landarbeitern und in ausgesprochen geringem Maße aus der Industriearbeiterschaft bzw. aus Jugendlichen, die von der Stadt aufs Land gingen. Sehr gezielt wurde staatlicherseits in die Rekrutierungsbedingungen und -mechanismen der Intelligenz eingegriffen, um eine Erweiterung der sozialen Basis durch die Förderung von Arbeiterkindern zu erreichen. Wenngleich in den fünfziger Jahren die wohl gravierendsten intra- und intergenerativen Strukturveränderungen zwischen den sozialen Hauptgruppen möglich waren, deuteten sich bereits gewisse Selbstrekrutierungstendenzen unter den Arbeitern und der Intelligenz an (vgl. Dittrich 1981, S. 262; Lötsch 1980, S. 99).

Die sich daran anschließende Stabilisierungsperiode in den sechziger Jahren brachte eine weitere Zunahme der Beschäftigten in hochqualifizierten Arbeiterberufen, der technischen Intelligenz und der Angestellten in den administrativen und dienstleistenden Bereichen mit sich. Ferner läßt sich in dieser Zeit ein drastischer Anstieg des Beschäftigunsgrades der Frauen feststellen, die in der Mehrzahl geringer qualifizierte Tätigkeiten im Dienstleistungssektor und der Industrie ausübten. Dagegen stagnierte und sank alsbald die Anzahl der in der Landwirtschaft Beschäftigten. Durch die Erweiterung von Bildungs- und Qualifikationsmöglichkeiten (mit der Einführung der zehnklassigen Oberschulbildung im Jahre 1965) wurde eine nahezu vollständige Eingliederung eines jeden Geburtsjahrganges in die Facharbeiterausbildung, Facharbeiter- und Teilfacharbeiterausbildung im Rahmen der Erwachsenenqualifizierung und eine außerordentlich rasche Zunahme der Studierenden an den Hoch- und Fachschulen erreicht.

Mit Beginn der siebziger Jahre setzte eine weitere Phase ein. Die mit der extensiven Strukturentwicklung im Zusammenhang stehenden sozialen Verschiebungen hatten sich nunmehr erschöpft. Es deuteten sich Grenzbedingungen in den Wachstumspotentialen der sozialen Gruppen an (vgl. Lötsch/Freitag 1981, S. 95), die im Verlaufe der siebziger Jahre zu Homogenisierungstendenzen und einer zunehmenden Uniformität in den Sozialprofilen führten. Die dysfunktionalen Wirkungen der Homogeni- 
sierungsbestrebungen wurden immer offensichtlicher, und zwar in dem Maße, in dem die Rezentralisierung der Volkswirtschaft weiter vorangetrieben worden ist. Dem daraus folgenden stetigen Innovationsrückgang versuche man staatlicherseits mit einem differenzierten Maßnahmenkatalog von Einkommensdifferenzierungen und abgestuften Privilegien zu begegnen und bestimmte soziale Gruppen in der Wissenschaft und unter den Arbeitern zu fördern (vgl. Wielgohs/Schulz 1990, S. 23). Gleichzeitig wurden vor allem aus beschäftigungspolitischen Erwägungen die Zulassungsquoten zum Hochschulstudium drastisch reduziert und die Aufnahmequoten für Abiturienten daran rigide angepaßt. Dadurch ist eine sich wechselseitig blockierende Entwicklungsdynamik im Verhältnis von Bildung und Beschäftigung in Gang gesetzt worden, die sowohl zu einschneidenden Begrenzungen sozialer Aufstiegschancen als auch zu erheblichen Dequalifizierungsprozessen in der Wirtschaft geführt hat. Der verstärkte administrative Eingriff in die Zuteilung von Bildungs- und Berufschancen beförderte die Stabilisierung und Schließung sozialer Reproduktionsquellen und wirkte sich somit als Rückgang an sozialen Veränderungsmöglichkeiten für die heranwachsende Generation aus (vgl. Kühnel 1987, S. 94). So sahen sich die Jugendlichen veranlaßt, die Wahl ihres Bildungs- und Berufsweges an eine zur Veränderung tendierende Sozialordnung anzupassen. Daß dabei die eh schon bestehenden sozialen Ungleichheiten im Generationswechsel erweitert reproduziert wurden, findet in den wenigen in der DDR durchgeführten Mobilitätsanalysen ihre Bestätigung.

Gerth (1982) untersuchte den Zusammenhang zwischen der sozialen Zugehörigkeit von Jugendlichen, die Ende der siebziger Jahre in den Arbeitsprozeß eintraten, und der sozialen Herkunft ihrer Eltern (die in der Regel in den vierziger und fünfziger Jahren ihre Arbeit aufnahmen) und ihren Großeltern (deren Eintritt in die Berufsarbeit um die Zeit des 1. Weltkrieges herum bzw. Anfang der zwanziger Jahren erfolgte). Die Befunde machen auf folgende Tendenzen aufmerksam: Während im Großeltern-ElternVergleich noch grundlegende Verschiebungen zwischen den sozialen Klassen und Schichten nachweisbar sind, vor allem in der Gruppe der Intelligenz, reproduzieren sich die sozialen Hauptgruppen im Eltern-Kinder-Vergleich überwiegend aus sich selbst. Jugendliche, der sozialen Herkunft nach Arbeiter, verbleiben überdurchschnittlich in dieser Position, wechseln leicht unterdurchschnittlich zu den Angestellten und beträchtlich unterdurchschnittlich in Intelligenzgruppen. Entstammen die Eltern hingegen den Angestellten oder der Intelligenz, dann schlagen die Heranwachsenden auch überdurchschnittlich eine intelligenz- oder angestelltentypische Laufbahn ein (vgl. Ebd. S. 178 f.). Eine andere Mobilitätsuntersuchung ist von Bathke (1985) vorgelegt worden. Er analysierte die Herkunftsbedingungen von Hochschulstudenten über jeweils zwei und drei Generationen. Unter Berücksichtigung der Qualifikation und beruflichen Tätigkeit der Eltern wurde festgestellt, daß sich Hochschulstudenten überproportional aus der Gruppe der Intelligenz und in politischer Hinsicht häufiger aus hochqualifizierten, in Partei und Staatsapparat tätigen Arbeitern und Angestellten rekrutieren. Jugendliche der sozialen Herkunft nach Facharbeiter sind hingegen im Hochschulstudium deutlich unterrepräsentiert (vgl. Bathke 1985, S. 35). Außerdem konnte Bathke nachweisen, daß es einen starken Zusammenhang zwi- 
schen der Fachrichtungswahl der Jugendlichen und dem Qualifikationsprofil der Eltern und Großeltern gibt (vgl. Ebd., S. 171).

Wenn die krisenhafte Entwicklung seit den siebziger Jahren von einem drastischen Rückgang an sozialen Veränderungsmöglichkeiten, wie auch von Schließungstendenzen in den Bildungs- und Berufswegen begleitet wurde, so bedeutet das jedoch nicht, daß es zu keinen Modernisierungen im Lebenszusammenhang von DDR-Jugendlichen gekommen ist. Mit dem Erreichen von Grenzbedinungen des sozialen Wachstums bei gleichzeitiger Verstärkung des staatlich-administrativen Steuerungsdrucks vollzog sich - gewissermaßen als Gegenbewegung - eine Aufwertung des Alltagskulturellen. Obgleich sich der Alltag doch am ehesten gesellschaftlichen Modernisierungen zu sperren scheint, bot er in begrenztem Maße, nicht zuletzt wegen der relativ geschlossenen Sozialordnung und einer zunehmenden Durchstaatlichung von Lebensbezügen, in der DDR, einen bevorzugten Raum für selbstorganisiertes Handeln und dem damit im Zusammenhang stehenden authentischen Erfahrungserwerb. Das gilt natürlich besonders für diejenigen Jugendlichen, die in dieser Krisenphase aufgewachsen sind und denen die eigene Zukunft als immer stärker zugebaut erscheinen mußte. So ist es offenbar zu gegenläufigen Entwicklungen im Lebenszusammenhang Heranwachsender gekommen. Während einerseits die schulische und vorberufliche Sozialisation einer immer stärkeren Standardisierung und Vorstrukturierung unterlag, gab es andererseits auch wiederum Anzeichen für eine Akzelleration und Ausdifferenzierung in den Erfahrungsbezügen und Lernfeldern nichtinstitutionalisierter Lebensbereiche.

\section{Soziokulturelle Segregationsprozesse zwischen den Generationen}

Wenn von Ausdifferenzierungs- und Individualisierungstendenzen unter den Jugendlichen in der DDR die Rede ist (vgl. Zentralinstitut für Jugendforschung 1990, S. 22), so erhielt diese Entwicklung doch einen anderen Zuschnitt als in kapitalistischen Gesellschaften (vgl. Beck 1986, S. 152). Enttraditionalisierung und Individualisierung waren in der DDR nicht die Folge von Flexibilisierungen in den Beschäftigungs- und Lebensverhältnissen, sondern ein Ergebnis des Anpassungsdrucks, der mit der zunehmenden Durchstaatlichung von Lebensbedingungen erzeugt wurde. Berufswahl, die Gestaltung des Lebensweges und politische Optionen sind einzig und allein dadurch zu risikovollen Unternehmen geworden, weil sie in mehr oder weniger starkem Maße den normativen Regelungen des staatlich-administrativen Systems in Wirtschaft, Politik und Ausbildung unterworfen werden mußten. Zwar waren innerhalb bestimmter Grenzen wohl individuelle Wahlhandlungen über die eigenen Lebensumstände möglich, keinesfalls jedoch gruppenspezifische Zusammenhänge und Identitäten in größerem Ausmaß herstellbar, die den Anspruch auf Selbstorganisation in nichtstaatlichen Infrastrukturen hätten geltend machen können. Insofern wird verständlich, daß die Familie als sozialer Rückzugsraum für die Jugendlichen eine große Rolle spielte. Trotz der hohen Scheidungsraten ${ }^{4}$ und der vielfach angezeigten Zerrüt- 
tungstendenzen ${ }^{5}$ weiß sich der überwiegende Teil der Jugendlichen im Vergleich zu anderen Sozialbezügen von den Eltern noch am gerechtesten behandelt (vgl. Soziale Erfahrungen der Schuljugend 1983, S. 158). In der Öffentlichkeit hingegen vermerken die Heranwachsenden eine eher distanzierte, von Voreingenommenheit geprägte Haltung der Erwachsenen ihnen gegenüber. In diesem Zusammenhang konzentrieren sich die Spannungen zur älteren Generation auf die Kritik an den »erwachsenengesteuerten « Institutionen und Räumen, die als hochgradig vorstrukturiert, anonym und distanziert erlebt werden. Typisch dafür ist die Aussage eines Jugendlichen, der seine Erfahrungen mit den Erwachsenen in der Öffentlichkeit in folgender Weise zum Ausdruck bracht:

"Jüngere Menschen -'so 30 bis 35jährige-haben vielleicht noch Verständnis, aber dann hörts schon auf... « Und: »Manchmal habe ich den Eindruck, daß die uns gar nicht verstehe wollen. Es gibt manchmal Situationen, da kommen sie sich so überlegen vor, da spürst du nicht die weitere Erfahrung. Es ist so, wenn wir mal unsre Meinung äußern, dann brechen sie das Gespräch ab oder gehen gar nicht darauf ein.« (Kollektive Erfahrungen. Vier Fallstudien. 1983)

Wohl am belastetsten haben die Jugendlichen ihre Beziehungen zur Erwachsenengeneration in der Schule erlebt. Hier traten ihnen die Lehrer vornehmlich als »Stundengeber « oder »Stoffeintrichterer « entgegen, wo sie in eine fast ausschließlich abhängige, reaktive und passive Rolle gedrängt wurden. So nimmt es auch nicht wunder, daß den Jugendlichen die Schule in starkem Maße in ihrer Disziplinierungsfunktion in Erinnerung geblieben ist.

Dennoch ist es unter der Decke des offiziellen Unterrichtsgeschehens auch zu subinstitutionellen Lern- und Verständigungsprozessen gekommen, wodurch die Jugendlichen Gruppensolidarität, Offenheit und Hilfsbereitschaft in der Bewältigung ihrer Lernanforderungen erfahren haben. Dieses Netzwerk an horizontalen Beziehungen hat das Leben in der Schule in einer bestimmten Weise auch wiederum erträglich werden lassen.

Obgleich sich die Heranwachsenden vor allem Beziehungen zu den Erwachsenen wünschten, die durch gegenseitige Achtung gekennzeichnet sind, sah die Realität dann doch ganz anders aus. In der Bewältigung alltäglicher Konflikte und Probleme blieben sie eher auf sich selbst verwiesen. Die an anderer Stelle beschriebenen Individualisierungsprozesse unter den Vorzeichen einer zunehmenden Verstaatlichung der Lebensverhältnisse haben offensichtlich zur Auseinanderentwicklung von Erfahrungszusammenhängen im Generationsverhältnis geführt. Im Alltag nahmen sie die Gestalt eines scheinbar konflitkfreien Aneinandervorbeilebens zwischen Jugendlichen und Erwachsenen an.

Aus bildungssoziologischen Untersuchungen (vgl. Soziale Erfahrungen 1983) geht zwar hervor, daß die Autoritätsverhältnisse zwischen den Generationen in Veränderung begriffen sind, sich neue kulturelle Suchbewegungen unter den Jugendlichen allerdings nur an den Rändern der von den Erwachsenenn verwalteten Organisationen und in den Nischen öffentlicher Räume wie im Privaten herausbilden konnten. Außer an einigen wenigen Orten in den Großstädten bestand kaum die Möglichkeit für eine selbstorganisierte alternative Infrastruktur in der DDR. Selbst die spektakulär gewor- 
denen Jugendkulturen und Szenen (vgl. Stock 1989) - eine Ausnahme bildeten die sich politisch engagierenden Gruppen unter dem Dach der Kirche (vgl. Kühnel/ Schulz/Wielghos 1990) - waren Phänomene einer ausgesprochenen Freizeitkultur. Der Ausstieg von Jugendlichen aus der Normalbiographie und der Einstieg in die nur marginalen alternativen Milieus traf nur für einen kleinen Kreis von ihnen zu. ${ }^{6}$ Man kann eher davon ausgehen, daß für den größten Teil der DDR-Jugendlichen eine $» n i-$ schenhafte « alltagskulturelle Modernisierung im privaten Bereich und an den Rändern öffentlicher Räume stattgefunden hat. Diese Entwicklung war hauptsächlich verbunden mit einem rezeptiven Mediengenuß von Rock- und Popmusik, Fernsehen, Diskothekenbesuchen und an die sozialen Bezüge in der Gruppe der Altersgleichen geknüpft (vgl. Voß 1981, S. 88). Als Teil der internationalen Kultur und als Medium, mit dem sich authentische Erfahrungen mit der DDR-Wirklichkeit recht gut transportieren ließen; spielte die Rockmusik eine herausragende Rolle. Auch wenn sie für den äußeren Betrachter das Signum des Hausgemachten und Provinziellen trug, kann man durchaus von einer hauseigenen Musikszene in der DDR sprechen. Wie der Text aus einem Song der Punkgruppe die »Skeptiker « deutlich macht, konnten die Jugendlichen in dieser Welt ihre Erlebnisse von Entfremdung, Ohnmacht und Widerstand am ehesten zum Ausdruck bringen:

»Immer treu und redlich sein,

dann kann auch nicht sehr viel geschehn.

Kräftemessende Gewalten, alles wirst Du überstehn.

Laß den Mantel windwärts flattern,

wieg dein Glück in Sicherheit, schneckenaufrecht ist dein Gang,

schon dreitausend Jahre lang

Refr.:

Kann der Wind sich drehn? Jaja!

Ins Gesicht ihm wehn? Jaja!

Kann Gefahr entstehn? Jaja!

Wird er windwärts gehen? Jajajaja!

Landparzelle und Kamickel, da erwacht die Seligkeit

Kindersegen, stille Liebe, ruhe sanft in Ewigkeit.

Friede, Freude, Eierkuchen, wer nicht lebt, bleibt immer tot.

In der Stickluft solcher Welten,

Mensch, da packt mich Atemnot.

Refr.:

Großes macht ihr für Kloaken, andres produziert ihr nicht.

Die Verrenkung eurer Seelen steht im feisten Angesicht.

Dummheit ist nicht totzukriegen,

spießig geht dic Welt zur Ruh,

alles Reden Magenwinde, Hoffnung stirbt bald völlig aus.

Refr.:

So viele wollen mit dem Arsch an die Wand,

Schleimer und Kriecher gibts in jedem Land.

Die erste Bürgexpflicht sind Ordnung und Ruh,

schlaft gut und macht die Augen nur fest zu.

Immer treu und redlich sein,

dann kann auch nicht sehr viel geschehn.

Kräftemessende Gewalten, alles wirst du überstehn. 
Laß den Mantel windwärts flattern,

wieg dein Glück in Sicherheit,

schneckenaufrecht ist dein Gang,

schon dreitausend Jahre lang.«

Eine solche Hereinnahme des Politischen in den Alltag stieß natürlich an Grenzen in Schule und Jugendorganisation.

Besonders kraß haben die in den achtziger Jahren heranwachsenden die Schizophrenie zwischen den offiziellen Normen und abgefragten Wissensbeständen und ihren eigenen authentischen Erfahrungen in der alltäglichen Auseinandersetzung mit einer problematischen Wirklichkeit erlebt (vgl. Soziale Erfahrungen 1983, S. 57). Dennoch war erstaunlich zu bemerken, wie mühelos die Jugendlichen mit dieser »Schranke im Kopf« die streng gezogenen Grenzen zwischen dem Offiziellen und der eigenen Erfahrungswelt zu »überspringen« schienen und damit umzugehen gelernt haben. Dieser Widerspruch wurde am nachhaltigsten von jenen Jugendlichen erlebt, die die besten Schulleistungen aufzuweisen und eine Funktion im Jugendverband innehatten. Dabei handelt es sich um Jugendliche, die sich in der Regel auch für einen weiterführenden Bildungsweg zur Hoch- oder Fachschule entschieden hatten und sich folglich auf die Modalitäten der politischen Kontrolle und Auslese in den höheren Bildungseinrichtungen einlassen mußten. Unter den Arbeiterjugendlichen sah das anders aus. Sofern sie nicht einen der besonders nachgefragten Berufe ergreifen wollten, brauchten sie sich nicht in dem Maße wie ihre aufstiegsorientierten Mitschüler auf das schizophrene Spiel nach den Regeln des Offiziellen einzulassen, konnten viel eher auf ihre authentischen Erfahrungen bauen. Im allgemeinen traten Jugendliche, die einen Facharbeiterberuf ergriffen, auch viel früher als andere aus der Jugendorganisation aus.

Die Tatsache, daß die politischen Erfahrungen der Heranwachsenden keinen Eingang in die Welt der Schule und der Jugendorganisation fanden, korrespondiert durchaus mit der Orientierung der Eltern, denen es in der Erziehung kaum auf Kritikfähigkeit und Offenheit im Umgang mit schulischen und politischen Fragen ankommt. In diesem Punkt bestand offenkundig ein Einverständnis zwischen den Generationen. Dennoch würden die Jugendlichen nicht soweit gehen, aus den Ansichten ihrer Eltern eine Art Leitbild für ihre eigene politische Urteilsfindung zu beziehen.

Daß eine soziokulturelle Differenzierung zwischen Erwachsenen und Jugendlichen Platz gegriffen hat, läßt sich ebenso an den unterschiedlichen sozialen Wahrnehmungsperspektiven der jeweils anderen Generation erkennen. Im allgemeinen zeigten sich die Eltern ziemlich unsicher in der Beurteilung der Erfahrungen ihrer Kinder. Sie scheinen die Erfahrungen, über die Jugendliche im Alter von 15 bis 16 Jahren verfügen, eher zu unterschätzen. Vor allem Eltern aus Arbeiterhaushalten waren der Auffassung, Jugendliche hätten noch keine eigenen Erfahrungen:

»Sie lemen heute viel Theorie, aber Erfahrungen - eigene-haben sie eigentlich kaum«, machte eine Mutter aus dem Arbeitermilieu geltend (Kollektive Erfahrungen. Vier Fallstudien 1983).

Offenkundig unterliegt das elterliche Wissen über die sozialen Erfahrungen ihrer Kinder einer sozialstrukturellen Spezifik. Es fiel auf, daß Angehörige der Intelligenz 
und leitende Angestellte den Jugendlichen eher Erfahrungen zutrauen als andere Elterngruppen. Diese Eltern waren auch in der Lage, die Quellen des Erfahrungserwerbs differenzierter und breiter zu sehen, ja sogar in Rechnung zu stellen, daß es unter den Jugendlichen einen »Erfahrungshunger « gibt. So äußerte ein Vater, daß »die Jugendlichen nicht nur zufällig Erfahrungen erwerben, sondern sie ja regelrecht suchen« (ebd.).

Bemerkenswert ist auch, wie stark die Geschlechterspezifik im Alltag der DDR-Familien immer noch durchschlägt. Zwar waren sich viele Eltern der Tatsache bewußt, daß man in der Erziehung von Jungen und Mädchen keine Unterschiede machen sollte, aber in der Realität gelten dann doch andere Maßstäbe. Eine Mutter äußerte sich darüber in folgender Weise.

»Anders anfassen muß man Mädchen eigentlich nicht als Jungen. Aber ich bin der Meinung, der Mann sollte doch noch ein bißchen höhergestellt sein, so beruflich. Sonst geht die Ehe nicht gut. Ich sage immer, als Frau muß3 man sich anpassen. Er stur und sie stur, das geht nicht.« (ebd.)

Ausgesprochen protektionistisch zeigten sich die Eltern gegenüber den Freizeitaktivitäten der Mädchen und andersgeschlechtlichen Freundschaftsbeziehungen. Dabei meinten sie zu wissen, daß ihre Kinder im allgemeinen für Liebesbeziehungen noch nicht reif seien und deshalb in der Regel noch über keine diesbezüglichen Erfahrungen verfügten. Generell schienen die Eltern den Reiz, den Mädchen und Jungen dieses Alters bereits aufeinander ausuiben und das große Interesse, das sie aneinander haben, zu unterschützen. Während das jeweils andere Geschlecht eines der Hauptgesprächsthemen in den Gruppen der Gleichaltrigen ist, gehörte es nicht zur familialen Kommunikation, daß über die aus ersten eigenen Versuchen, selbst erlebten Erfolgen und Enttäuschungen gewonnenen Erfahrungen über den Umgang mit dem anderen Geschlecht, Sexualität, auch Ängste und Hemmungen gesprochen wird. Im Gegenteil, meistenteils sorgten die Eltern für eine Tabuisierung dieser Erfahrungen, ihrer eigenen darin eingeschlossen (vgl. Nickel 1983, S. 168).

Wenn die Identitässuche der Jugendlichen in den achtziger Jahren andere Wege nimmt als die ihrer Eltern, so hat das vor allem etwas mit dem veränderten Bezug zu Arbeit und Beruf zu tun. Von nahezu allen Jugendlichen wurde die Sicherheit des künftigen Arbeitsplatzes nicht angezweifelt, aber nur für eine Minderheit ging der im letzten Schuljahr angestrebte Berufswunsch auch wirklich in Erfüllung. ${ }^{\text {? }}$

Restriktive Zulassungsbestimmungen und Schließungsprozesse auf seiten des Beschäftigtensystems begünstigten eine inflationäre Entwicklung von Leistungsnachweisen im Verhältnis zu den realen Berufschancen. Recht treffend bringt das ein Jugendlicher auf den Punkt:

»Ja, ja, das war früher leichter, eine gute Lehrstelle zu bekommen. Jetzt braucht man immer bessere Zensuren, sonst ist man angeschissen « (Ebd.)

Wurde diese Problemsicht vorzugsweise von den Schülern vorgebracht, so schienen die Eltern hingegen sorgloser zu sein. Am optimistischsten zeigten sich Eltern mit einem Hoch- und Fachschulabschluß, deren Kinder die vergleichsweise besten schulischen Leistungen aufzuweisen hatten.

So gab es Eltern, die auf die Frage nach den Berufschancen ihrer Kinder einen Opti- 
mismus durchscheinen ließen, der offenbar an die eigene Aufbaustimmung der fünfziger Jahre erinnert:

$\gg$ Jeder ist seines Glückes Schmied. Wenn jemand ehrlich und ordentlich ist, macht er seinen Weg schon.« (ebd.)

Daß die künftigen Entwicklungschancen nicht im Ermessen des einzelnen liegen, dürfte wahrscheinlich zum soliden Marschgepäck der Jugendlichen in der DDR gehören, den sie über die sog. »Wende« mit hinübergerettet haben. Möglicherweise wird es gerade diese Generation sein, die, um mit Mannheim zu argumentieren, der gegenwärtigen Problematik wohl am nächsten ist (vgl. Mannheim 1970, S. 539, 540) und die soziokulturelle Integration in eine Vereinigte Bundesrepublik Deutschland am schnellsten bewältigt. Und zwar einfach deshalb, weil die Jugendlichen der achtziger Jahre nie eine solche gesellschaftliche Verwurzelung erfahren haben wie das ihre Eltern erlebten. Annehmen läßt sich, daß die Segregationsprozesse zwischen den Generationen mit der Modernisierung noch weiter durchgreifen werden, wobei die Erfahrungs- und Lerndefizite wahrscheinlich am stärksten bei den älteren Generationen zu verbuchen sind, die traditionellen soziokulturellen Milieus entstammen.

\section{Anmerkungen}

1 Zweifellos gibt es eine ganze Reihe von ernstzunehmenden Einwänden gegen das Mannheimsche Generationskonzept, beispielsweise bezüglich der Parallelisierung von Klassen- und Generationsbegriff oder die unbegründete Annahme homogener Lebenszusammenhänge aufgrund einer gleichartigen Verarbeitung zeitgeschichtlicher Bedingungen. Angesichts der Veränderungen in der Biographie Jugendlicher muß man sich auch fragen, wie fest strukturiert eine »formative Phase« heutzutage noch sein kann.

2 Dabei handelt es sichausschließlich um Mobilitätsuntersuchungen. Die einzigen, die in der DDR dazu bislang vorgelegt wurden, stammen von Gerth (1982), Bathke (1985) und Lötsch/Freitag (1981).

3 Diesbezügliche Untersuchungen sind vor allem am Zentralinstitut für Jugendforschung angestellt worden. Sie beruhen im allgemeinen auf der Messung von Einstellungen. Vgl. dazu Friedrich (1990).

4 In einer Vorlage zur Beratung des Runden Tisches »Jugend« vom 5. März 1990 wurde darauf aufmerksam gemacht, daß »die in den 70er Jahren geschlossenen Ehen (relevant für die Jugend der 80er Jahre)... nur zu ca. 75 Prozent Bestand (haben), und für die in den $80 \mathrm{er}$ Jahren geschlossenen Ehen gilt dies für 60 Prozent. Gegenwärtig leben etwa 15 Prozent aller Jugendlichen bei der alleinstehenden Mutter, weitere 15 Prozent haben einen Stiefelternteil (13 Prozent Stiefväter).«(Vgl.davon Ausgewählte Zahlen und Fakten zur Lage der Kinder und Jugendlichen in der DDR (1990, S.44)

5 Hinweise dafür finden sich in einer Studie des Zentralinstituts für Jugendforschung Problempapier Jugend (1990, S. 24).

6 Man rechnet etwa mit 10 Prozent der Jugendlichen in der DDR, die in der Vergangenheit der subkulturellen Szene angehörten.

7 Lediglich für knapp ein Drittel der Jugendlichen eines Altersjahrganges hat sich der Berufswunsch erfüllen lassen. Vgl. Kühnel (1990).

\section{Literatur}

Adamski, WI. (1982): Structural and Generational Aspects of a Social Conflict, in. Polish Academy of Sciences, PWN - Polish Scientific Publishers (ed.): Sisyphus. Sociological Studies, Warsaw.

Ausgewählte Zahlen und Fakten zur Lage der Kinder und Jugendlichen in der DDR (1990). Vorlage zur Beratung des Rundes Tisches, Ms., Berlin. 
Bathke, G.W. (1985): Sozialstrukturelle Herkunftsbedingungen und Persönlichkeitsentwicklung von Studenten, Diss., Berlin-Leipzig.

Beck, U. (1986): Risikogesellschaft. Auf dem Weg in eine andere Modeme, Frankfurt a.M.

Dittrich, G. (1981): Zu den Reproduktionsquellen und einigen Veränderungen in der Struktur der Arbeiterklasse der DDR während der Übergangsperiode vom Kapitalismus zum Sozialismus (1945 bis 1961), in: Akademie der Wissenschaften der DDR (Hrsg.): Jahrbuch für Wirtschaftsgeschichte, Teil II, Berlin.

Friedrich, W. (1990): Mentalitätswandlungen der Jugend in der DDR, in: Aus Politik und Zeitgeschichte . Beilage zur Wochenzeitschrift Das Parlament, B 16-17.

Fuchs, W. (1986): Jugend der 50er Jahre und Jugend der 80er Jahre - Vergleich und Verhältnis, In: Remschmidt, H. (Hrsg.): Jugend und Gesellschaft, Realitätsbewältigung, Krisen und Auswege, StuttgartFrankfurt.

Gerth, W. (1982): Persönlichkeitsentwicklung und sozialstrukturelle Bedingungen der Denk- undVerhaltensentwicklung junger Werktätiger, Diss., Berlin.

Kollektive Erfahrungen. Vier Fallstudien (1983), Akademie der Pädagogischen Wissenschaften der DDR, Berlin.

Kühnel, W. (1987): Jugend in den gesellschaftlichen Strukturveränderungen. Ein Beitrag zur generationssoziologischen Analyse des Lebenszusammenhanges Heranwachsender. Diss., Berlin.

Kühnel, W. (1990): Der Lebenszusammenhang DDR-Jugendlicher im Spannungsfeld von institutioneller Verregelung und alltagskultureller Modernisierung, in: Sozialisation im Sozialismus. Sonderheft der Zeitschrift für Sozialisationsforschung.

Kühnel, W./Schulz, M./Wielgohs, J. (1990): Vom politischen Protest zur parlamentarischen Interessenvertretung. Soziale Bewegungen im Umbruch der DDR-Gesellschaft, in:Zeitschrift für Parlamentsfragen, Heft 1 .

Lötsch, M. (1980): Zur Entwicklung der Intelligenz in der Deutschen Demokratischen Republik, in: Autorenkolletiv: Die Intelligenz in der sozialistischen Gesellschaft, Berlin.

Lötsch, M./Freitag, J. (1981): Sozialstruktur und Mobilität, in: Akademie der Wissenschaften der DDR (Hrsg.): Jahrbuch für Soziologie und Sozialpolitik, Berlin.

Mannheim, K. (1970): Das Problem der Generationen, in: ders.: Wissenssoziologie. Auswahl aus dem Werk, Neuwied am Rhein und Berlin.

Matthes, U./Müller-Hartmann, I. (1990): Zur politischen Stimmungslage der DDR-Bevölkerung im Zeitraum November 1988 bis November 1989, Ms., Berlin.

Mayer, K.U. (1980): Gesellschaftlicher Wandel und soziale Struktur des Lebensverlaufs. Arbeitspapier Nr. 38, Referat auf dem 20. Deutschen Soziologentag, Bremen.

Mitter, A./Wolle, St. (1990): Ich liebe Euch doch alle! Befehle und Lageberichte des MfS, Berlin.

Mühler, K./Wilsdorf, St.H. (1990): Die Leipziger Montagsdemonstration-Aufstieg und Wandel einer basisdemokratischen Institution des friedlichen Umbruchs im Spiegel empirischer Meinungsforschung, Karl-Marx-Universität, Institut für Politikwissenschaft und Soziologie, Ms., Leipzig.

Nickel, H.M. (1983): Alltagserfahrungen im Generationsverhältnis, in: Soziale Erfahrungen der Schuljugend. Akademie der Pädagogischen Wissenschaften, Berlin.

Schelsky, H. (1957): Die skeptische Generation, Düsseldorf-Köln.

Soziale Erfahrungen der Schuljugend (1983), Akademie der Pädagogischen Wissenschaften der DDR, Berlin.

Stock, M. (1990): Zur Soziologie gegenwärtiger Jugendkulturen in der DDR - ein theoretisch-konzeptioneller Ansatz und empirische Befunde, Diss. Berlin.

Voß, P. (1981): Die Freizeit der Jugend, Berlin.

Wielgohs, J./Schulz, M. (1990): Reformbewegung und Volksbewegung. Politische und soziale Aspekte im Umbruch der DDR-Gesellschaft, in: Aus Politik und Zeitgeschichte. Beilage zur Wochenzeitschrift Das Parlament, B16-17.

Zentralinstitut für Jugendforschung (1990): Problempapier Jugend Leipzig, Ms. 